\title{
Rotor Speed and Parameters Identification Scheme for Sensorless Induction Motor Drives
}

\author{
Djamila Cherifi", Yahia Miloud \\ Electrical Engineering Department, Faculty of technology, Dr Moulay Tahar University, Saida, Algeria
}

Email address:

d_cherifi@yahoo.fr (D. Cherifi)

${ }^{*}$ Corresponding author

\section{To cite this article:}

Djamila Cherifi, Yahia Miloud. Rotor Speed and Parameters Identification Scheme for Sensorless Induction Motor Drives. Science Journal of Circuits, Systems and Signal Processing. Vol. 6, No. 6, 2017, pp. 57-64. doi: 10.11648/j.cssp.20170606.12

Received: December 15, 2017; Accepted: December 28, 2017; Published: January 18, 2018

\begin{abstract}
Most industrial processes have time-varying parameters, the Induction Motor IM is a typical example, in fact the rotor resistance (Rr) can vary and reach $100 \%$ of its nominal value during operation because of the heating of the rotor, the effect of the variation of the stator resistance (Rs) is very influential at low speed for our case because it can go up to $50 \%$ of its initial value, it is proposed in this work to developed a method that involves the observation of the speed and the simultaneous estimation of the principal parameters varying in time, and in particular the estimation of the stator resistance Rs and rotor $\mathrm{Rr}$ of the induction motor using sliding-mode observer when only the stator currents and voltages are accessible by measurement. After the theoretical study we will validate the method proposed by the simulation results where we will show the fairly fast convergence of this method as well as its robustness.
\end{abstract}

Keywords: IM Control, Sliding Mode Observer, Parameter Estimation, Field Oriented Controller

\section{Introduction}

The field-oriented control technique has been widely used for high-performance induction motor (IM) drive With this technique, the decoupling of torque and flux control commands of the induction motors is guaranteed and the induction motor can be controlled linearly as a separated excited dc motor. Indirect field-oriented control (IFOC) is one of the most effective vector controls of IM due to the simplicity of design and implementation [1]. But, the knowledge of the rotor speed is necessary, this necessity requires additional speed sensor which adds to the cost and the complexity of the drive system. Over the past few years, ongoing research has concentrated on the elimination of the speed sensor at the machine shaft without deteriorating the dynamic performance of the drive control system. The advantages of speed sensorless induction motor drives are reduced hardware complexity and lower cost, reduces size of the drive machine, elimination of the sensor cable, better noise immunity, increased reliability and less maintenance requirements, [2].

In order to achieve good performance of sensorless vector control, different speed estimation schemes have been proposed, and a variety of speed estimators exist nowdays [3]. Such as direct calculation method, model reference adaptive system (MRAS), Extended Kalman Filters (EKF), Extended Luenberger observer (ELO), ect.

Out of various approaches, Luenberger observer based speed sensorless estimation has been recently used, due to its good performance and case of implementation. The Luenberger observer (LO) belongs to the group of closed loop observers. It is a deterministic type of observer because it is based on a deterministic model of the system, [4].

Therefore, parameter errors can degrade the speed control performance. However, the rotor and the stator resistance variation has a great influence on the speed estimation [2], [5]. So, online adaptation of the stator resistance can improve the performance of sensorless IFOC drive at the low speed region. So, a simultaneous estimation of rotor speed with stator and stator resistance is presented based on a luenberger observer.

This paper is organized as follows. In Section 2, dynamic model of induction motor is reported; principle of fieldoriented controller is given in Section 3. The proposed solution is presented in Section 4. In Section 5, results of 
simulation tests are reported. Finally, Section 6 draws conclusions.

\section{Dynamic Model of Induction Motor}

By referring to a rotating reference frame, denoted by the superscript $(d, q)$, the dynamic model of a three-phase induction motor can be expressed as follows, [2]:

$$
\left\{\begin{array}{l}
\frac{d}{d t} i_{d s}=-A_{1} \cdot i_{s d}+\omega_{s} i_{s q}+\frac{L_{m}}{\sigma \cdot L_{s} \cdot L_{r} \cdot T_{r}} \cdot \varphi_{r d}+A_{2} \omega_{r} \varphi_{r q}+A_{3} \cdot V_{s d} \\
\frac{d}{d t} i_{q s}=-\omega_{s} i_{s d}-A_{1} \cdot i_{s q}-A_{2} \omega_{r} \varphi_{r d}+\frac{L_{m}}{\sigma \cdot L_{r} \cdot L_{s} \cdot T_{r}} \cdot \varphi_{r q}+A_{3} \cdot V_{s q} \\
\frac{d}{d t} \varphi_{d r}=\frac{L_{m}}{T_{r}} i_{s d}-\frac{1}{T_{r}} \varphi_{r d}+\left(\omega_{s}-\omega_{r}\right) \cdot \varphi_{r q} \\
\frac{d}{d t} \varphi_{q r}=\frac{L_{m}}{T_{r}} \cdot i_{s q}-\left(\omega_{s}-\omega_{r}\right) \cdot \varphi_{r d}-\frac{1}{T_{r}} \varphi_{r q} \\
\frac{d \omega_{r}}{d t}=\frac{p}{J}\left(T_{e m}-T_{l}\right)-\frac{f}{J} \omega_{r}
\end{array}\right.
$$

Where

$$
\begin{aligned}
& A_{1}=\left(\frac{R_{s}}{\sigma \cdot L_{s}}+\frac{1-\sigma}{\sigma \cdot T_{r}}\right) ; A_{2}=\frac{L_{m}}{\sigma \cdot L_{s} \cdot L_{r}} \\
& A_{3}=\frac{1}{\sigma \cdot L_{s}} ; \sigma=1-\frac{L_{m}^{2}}{L_{s} L_{r}} ; \omega_{g}=\omega_{s}-\omega_{r} \\
& T_{e m}=\frac{3}{2} P \frac{L_{m}}{L_{r}}\left(\varphi_{\mathrm{rd}} \cdot \mathrm{i}_{\mathrm{sq}}-\varphi_{\mathrm{rq}} \cdot \mathrm{i}_{\mathrm{sd}}\right)
\end{aligned}
$$

$\omega_{s}$ and $\omega_{r}$ are the electrical synchronous stator and rotor speed; $\sigma$ is the linkage coefficient, and $T_{r}$ is the rotor time constants.

\section{Principle of Field Oriented Controller (Foc)}

There are tow categories of vector control strategy. We are interested in this study to the so-called IFOC. As shows in Eq (1) that the expression of the electromagnetic torque in the dynamic regime presents a coupling between stator current and rotor flux, [2].

The main objective of the vector control of induction motors is, as in DC machines, to independently control the torque and the flux; this is done by using a $d-q$ rotating reference frame synchronously with the rotor flux space vector. The d-axis is then aligned with the rotor flux space vector (Blaschke, 1972). Under this condition we get:

$$
\varphi_{\mathrm{rd}}=\varphi_{\mathrm{r}} \text { and } \varphi_{\mathrm{rq}}=0
$$

The torque equation becomes analogous to the DC machine and can be described as follows:

$$
T_{e}=\frac{3}{2} p \frac{L_{m}}{L_{r}}\left(\varphi_{r} i_{s q}\right)
$$

It is right to adjust the flux while acting on the stator current component $i_{\text {sd }}$ and to adjust the torque while acting on the $i_{\text {sq }}$ component.

Using the Eq (1) we get:

$$
\begin{gathered}
i_{s d}=p \frac{\left(1+T_{r} s\right)}{L_{m}} \varphi_{r}^{\bullet} \\
i_{s q}=\frac{T_{r}}{L_{m}} \omega_{g l}^{\bullet} \varphi_{r}^{\bullet}
\end{gathered}
$$

We replace $i_{s q}$ by its expression to obtain $T_{e}$ as function of the reference slip speed $\omega_{g l}^{\bullet}$

$$
T_{e}=\frac{3}{2} p \frac{\varphi_{r}^{\bullet 2}}{R_{r}} \omega_{g l}^{\bullet}
$$

The stator voltage commands are:

$$
\left\{\begin{aligned}
v_{s d} & =R_{s} i_{s d}-\sigma L_{s} \omega_{s} i_{s q}+\sigma L_{s} \frac{d i_{s d}}{d t}+\frac{L_{m}}{L_{r}} \frac{d \varphi_{r}}{d t} \\
& =v_{s d 1}-\omega_{s} \cdot \sigma \cdot L_{S} \cdot i_{S q} \\
v_{s q} & =R_{s} i_{s q}+\sigma L_{s} \omega_{s} i_{s d}+\sigma L_{s} \frac{d i_{s q}}{d t}+\frac{L_{m}}{L_{r}} \omega_{s} \varphi_{r} \\
& =v_{s q 1}-\omega_{s} \cdot \sigma \cdot L_{S} \cdot i_{S d}-\frac{L_{m}}{L_{r}} \cdot \omega \varphi_{r}
\end{aligned}\right.
$$

The voltages $\mathrm{v}_{\mathrm{sd}}$ and $\mathrm{v}_{\mathrm{sq}}$ should act on the current $\mathrm{i}_{\mathrm{sd}}$ and $\mathrm{i}_{\mathrm{sq}}$ separately and consequently the flux and the torque. The twophase stators current are controlled by two PI controllers taking as input the reference values $i_{\text {sd }}^{\bullet}, \dot{i}_{\mathrm{sq}}^{\bullet}$ and the measured values. Thus, the common thought is to realize the decoupling by adding the compensation terms ( $\tilde{e}_{s d}$ and $\left.\tilde{e}_{s q}\right)$.

The block decoupling is described by the following equations:

$$
\begin{aligned}
& \tilde{e}_{s d}=\omega_{s} \cdot \sigma \cdot L_{S} \cdot i_{S q} \\
& \tilde{e}_{s q}=-\omega_{s} \cdot \sigma \cdot L_{S} \cdot i_{S d}-\frac{L_{m}}{L_{r}} \cdot \omega \varphi_{r}
\end{aligned}
$$

It is necessary to determine the amplitude and the position of rotor flux. In the case of an indirect field oriented control, the module is obtained by a block of field weakening given by the following non linear relation:

$$
\varphi_{r}^{\bullet}= \begin{cases}\varphi_{r n} & \text { if }\left|\omega_{r}\right| \leq \omega_{r n} \\ \varphi_{r n} \frac{\omega_{r n}}{\left|\omega_{r}\right|} & \text { if }\left|\omega_{r}\right|>\omega_{r n}\end{cases}
$$

The slip frequency can be calculated from the values of the 
stator current quadrate and the rotor flux oriented reference frame as follow:

$$
\begin{aligned}
\omega_{g} & =\omega_{s}-\omega_{r} \\
& =\frac{L_{m}}{T_{r}} \cdot \frac{i_{S q}}{\varphi_{r d}}=\frac{1}{T_{r}} \frac{i_{s q}}{i_{s d}}
\end{aligned}
$$

The rotor flux position is given by:

$$
\theta_{S}=\int \omega_{S} \cdot d t=\int\left(p . \Omega+\frac{L_{m} \cdot i_{S q}}{T_{R} \cdot \varphi_{r}^{\bullet}}\right) \cdot d t
$$

For Speed Regulation, the use of a classical PI controller makes appear in the closed loop transfer function a zero, which can influence the transient of the speed. Therefore, it is more convenient to use the so-called IP controller which has some advantages as a tiny overshoot in its step tracking response, good regulation characteristics compared to the proportional plus integral (PI) controller and a zero steadystate error, [2].

$$
\frac{\omega_{r}(s)}{\omega_{r}^{*}(s)}=\frac{k_{i} \cdot k_{p} \cdot k_{t} \cdot p}{J \cdot s^{2}+\left(B+k_{p} \cdot k_{t} \cdot p\right) \cdot s+k_{i} \cdot k_{p} \cdot k_{t} \cdot p}
$$

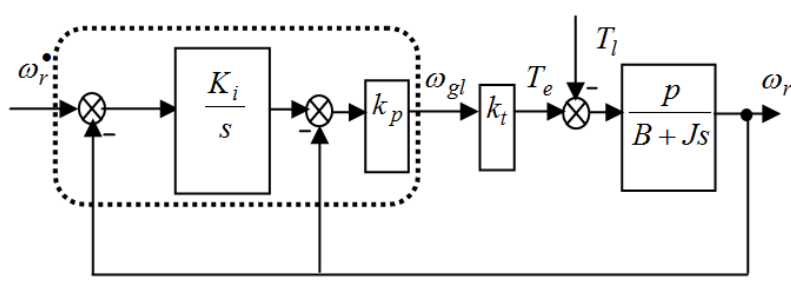

Figure 1. Bloc diagram of IP speed controller.

The gains of IP controller, $\mathrm{K}_{\mathrm{p}}$ and $\mathrm{K}_{\mathrm{i}}$, are determined using a design method to obtain a trajectory of speed with the desired parameters ( $\xi$ and $\omega_{n}$ ). The gains parameters values of the IP speed controller are easily obtained as:

$$
\left\{\begin{array}{l}
K_{p \omega}=\frac{\left(2 . \xi . \omega_{n} \cdot J-B\right) R_{r}}{P \cdot \varphi_{r}^{2}} \\
K_{i \omega}=\frac{J \cdot \omega_{n}^{2}}{K_{p \omega} \cdot p^{2} \cdot \varphi_{r}^{2}}
\end{array}\right.
$$

\section{Luenberger Observer}

The Luenberger observer (LO) belongs to the group of closed loop observers. It is a deterministic type of observer because it is based on a deterministic model of the system. This observer can reconstruct the state of a system observable from the measurement of inputs and outputs. It is used when all or part of the state vector can not be measured, [2], [7].

It allows the estimation of unknown parameters or variables of a system.

The equation of the Luenberger observer can be expressed as:

$$
\left\{\begin{array}{l}
\tilde{\dot{X}}=A \tilde{X}+B U+K(Y-\tilde{Y}) \\
\tilde{Y}=C \tilde{X}
\end{array}\right.
$$

In this work, a sensorless Indirect Rotor-Flux-oriented Control (IFOC) of induction motor drives is studied. The strategy to estimate the rotor speed, stator resistance and the flux components is based on Luenberger state-observer (LO) including an adaptive mechanism based on the lyaponov theory, as displayed in Figure 3.

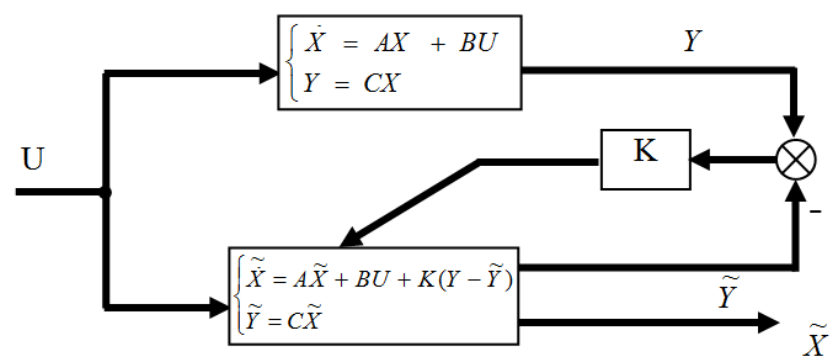

Figure 2. Luenberger Observer.

\subsection{Rotor Model of Induction Motor in the Coordinate $(\alpha, \beta)$}

The model of the induction motor can be described by following state equations in the stationary reference $(\alpha, \beta)$, [2], [8]:

$$
\left\{\begin{array}{l}
\hat{X}=A \cdot X+B . U \\
Y=C \cdot X
\end{array}\right.
$$

With: $\quad X=\left[\begin{array}{llll}i_{s \alpha} & i_{s \beta} & \varphi_{r \alpha} & \varphi_{r \beta}\end{array}\right]^{T} ; \quad U=\left[\begin{array}{ll}v_{s \alpha} & v_{s \beta}\end{array}\right]^{T} ;$ $Y=\left[\begin{array}{ll}i_{s \alpha} & i_{s \beta}\end{array}\right]^{T}$

The state equations can be written as follows:

$$
\left\{\begin{array}{l}
\hat{i}_{s \alpha}=a_{1} \cdot i_{s \alpha}+a_{2} \cdot \varphi_{r \alpha}-a_{3} \cdot \omega_{r} \cdot \varphi_{r \alpha}+a_{6} \cdot v_{s \alpha} \\
\hat{i}_{s \beta}=a_{1} \cdot i_{s \beta}+a_{2} \cdot \varphi_{r \beta}+a_{3} \cdot \omega_{r} \cdot \varphi_{r \alpha}+a_{6} \cdot v_{s \beta} \\
\hat{\varphi}_{r \alpha}=a_{4} \cdot i_{s \alpha}+a_{5} \cdot \varphi_{r \alpha}-\omega_{r} \cdot \varphi_{r \beta} \\
\hat{\varphi}_{r \beta}=a_{4} \cdot i_{s \beta}+a_{5} \cdot \varphi_{r \beta}+\omega_{r} \cdot \varphi_{r \alpha}
\end{array}\right.
$$

Where:

$$
\begin{gathered}
a_{1}=-\frac{1}{\sigma \cdot T_{s}}-\frac{(1-\sigma)}{\sigma \cdot T_{r}} ; a_{2}=\frac{L_{m}}{\sigma \cdot L_{s} \cdot L_{r}} \cdot \frac{1}{T_{r}} ; a_{3}=-\frac{L_{m}}{\sigma \cdot L_{s} \cdot L_{r}} \\
a_{4}=\frac{L_{m}}{T_{r}} ; a_{5}=-\frac{1}{T_{r}} ; a_{6}=\frac{1}{\sigma \cdot L_{s}} .
\end{gathered}
$$

\subsection{Determination of the Gain Matrix}

The determination of the matrix $\mathrm{K}$ using the conventional procedure of pole placement. We proceed by imposing the poles of the observer and therefore it's dynamic. 
Determining the coefficients of $\mathrm{K}$ by comparing the characteristic equation of the observer with the one we wish to impose. In developing the different matrices $\mathrm{A}, \mathrm{C}$ and $\mathrm{K}$ we obtain the following equation:

$$
\begin{gathered}
p^{2}+\left(\frac{1}{\sigma \cdot T_{S}}+\frac{1}{\sigma \cdot T_{r}}-j \hat{\omega}_{r}+K^{\prime}\right) p+\left(\frac{1}{T_{r}}-j \hat{\omega}_{r}\right)\left\{\left(\frac{1}{\sigma \cdot T_{S}}+\frac{1}{\sigma \cdot T_{r}}\right)+K^{\prime}\right\}+ \\
+\left(\frac{L_{m}}{T_{r}}-K^{\prime \prime}\right)\left(\frac{L_{m}}{\sigma \cdot L_{S} \cdot L_{r}}\right)\left(\frac{1}{T_{r}}-j \hat{\omega}_{r}\right)=0
\end{gathered}
$$

Or K' and K" are complex gains.

The dynamics of the observer is defined by the following equation:

$$
\begin{gathered}
p^{2}+k \cdot\left(\frac{1}{\sigma \cdot T_{s}}+\frac{1}{\sigma \cdot T_{r}}-j \hat{\omega_{r}}\right) p+k^{2} \cdot\left(\frac{1}{T_{r}}-j \hat{\omega_{r}}\right)\left\{\left(\frac{1}{\sigma \cdot T_{s}}+\frac{1}{\sigma \cdot T_{r}}\right)\right\}+ \\
+\left(\frac{L_{m}}{T_{r}}\right)\left(\frac{L_{m}}{\sigma \cdot L_{s} \cdot L_{r}}\right)\left(\frac{1}{T_{r}}-j \hat{\omega}_{r}\right)=0
\end{gathered}
$$

Whose roots are proportional to the poles of the MAS; the proportionality constant $\mathrm{k}$ is at least equal to unity $(0<\mathrm{k}>1)$

The identification of expressions (16) and (17) gives:

$$
\begin{gathered}
K^{\prime}=(k-1) \cdot\left(\frac{1}{\sigma \cdot T_{S}}+\frac{1}{\sigma \cdot T_{r}}-j \hat{\omega} r\right) \\
K^{\prime \prime}=(k-1) \cdot\left\{\mid\left\{\frac{1}{\sigma \cdot T_{s}}+\frac{1}{\sigma \cdot T_{r}}\right] \cdot \frac{\sigma \cdot L_{s} \cdot L_{m}}{L_{r}}-\frac{L_{m}}{T_{r}}\right\}(k-1) \\
-\frac{\sigma \cdot L_{s} \cdot L_{m}}{L_{r}}\left[\frac{1}{\sigma \cdot T_{s}}+\frac{1}{\sigma \cdot T_{r}}\right]+j \hat{\omega}_{r} \frac{\sigma \cdot L_{s} \cdot L_{m}}{L_{r}} \mid
\end{gathered}
$$

For the coefficients of the gain matrix of the observer is placed:

$$
\begin{aligned}
& K^{\prime}=K_{1}+j K_{2} \\
& K^{\prime \prime}=K_{3}+j K_{4}
\end{aligned}
$$

and in accordance with the antisymmetry of the matrix A we set the gain as follows:

$$
K=\left[\begin{array}{cc}
K_{1} & -K_{2} \\
K_{2} & K_{1} \\
K_{3} & -K_{4} \\
K_{4} & K_{3}
\end{array}\right]
$$

Or:

$$
\left\{\begin{array}{c}
K_{1}=(k-1) \cdot\left(\frac{1}{\sigma \cdot T_{S}}+\frac{(1-\sigma)}{\sigma \cdot T_{r}}+\frac{1}{T_{r}}\right) \\
K_{2}=(k-1) \cdot \hat{\omega} r \\
K_{3}=\frac{\left(1-k^{2}\right)}{a_{3}} \cdot\left(\frac{1}{\sigma \cdot L_{S}}+\frac{(1-\sigma)}{\sigma \cdot T_{r}}+\frac{a_{3}}{T_{r}}\right)+ \\
\quad+\frac{(k-1)}{a_{3}} \cdot\left(\frac{1}{\sigma \cdot T_{S}}+\frac{(1-\sigma)}{\sigma \cdot T_{r}}+\frac{1}{T_{r}}\right) \\
K_{4}=\frac{(k-1)}{a_{3}} \cdot \hat{\omega}_{r}
\end{array}\right.
$$

The poles of the observer are chosen to accelerate convergence to the dynamics of the open loop system. In In general, the poles are 5-6 times faster, but they must remain slow compared to measurement noise, so that we choose the constant k usually small.

\subsection{State Representation of the Luenberger Observer}

As the state is generally not available, the goal of an observer is to place an order by state feedback and estimate this state by a variable which we denote $\hat{X}$ :

Where:

$$
\hat{X}=\left[\begin{array}{llll}
\hat{I}_{s \alpha} & \hat{I}_{s \beta} & \hat{\varphi}_{r \alpha} & \hat{\varphi}_{r \beta}
\end{array}\right]^{T}
$$

So the state space of the observer becomes as follows:

$$
\hat{\dot{X}}=A_{\omega_{r}}\left(\hat{\omega}_{r}\right) \cdot \hat{X}+B \cdot U+K .\left(I_{s}-\hat{I}_{s}\right)
$$

With

$$
\left(I_{s}-\hat{I}_{s}\right)=\left[\begin{array}{ll}
I_{s \alpha}-\hat{I}_{s} & I_{s \beta}-\hat{I}_{s}
\end{array}\right]
$$

\subsection{Adaptive Luenberger Observer for Speed Estimation}

Suppose now that speed is an unknown constant parameter. It's about finding an adaptation law that allows us to estimate it. The observer can be written, [2]:

$$
\hat{\dot{X}}=A_{\omega_{r}}\left(\hat{\omega}_{r}\right) \cdot \hat{X}+B \cdot U+K \cdot\left(I_{S}-\hat{I}_{S}\right)
$$

With

$$
A_{\omega_{r}}\left(\hat{\omega_{r}}\right)=\left[\begin{array}{cccc}
a_{1} & 0 & a_{2} & -a_{3} \cdot \hat{\omega} \\
0 & a_{1} & -a_{3} . \hat{\omega} & a_{2} \\
a_{4} & 0 & a_{5} & -\hat{\omega}_{r} \\
0 & a_{4} & \hat{\omega}_{r} & a_{5}
\end{array}\right]
$$

The mechanism of adaptation speed will be reduced by Lyapunov theory. The estimation error of the stator current and rotor flux, which is simply the difference between the observer and the engine model, is given by:

$$
\dot{e}=(A-K . C) \cdot e+(\Delta A) \cdot \hat{X}
$$

With

$$
\Delta A=A\left(\omega_{r}\right)-A\left(\hat{\omega}_{r}\right)=\left[\begin{array}{cccc}
0 & 0 & 0 & a_{3} \Delta \omega_{r} \\
0 & 0 & -a_{3} \Delta \omega_{r} & 0 \\
0 & 0 & 0 & -\Delta \omega_{r} \\
0 & 0 & \Delta \omega_{r} & 0
\end{array}\right]
$$

Or: $\Delta \omega_{r}=\omega_{r}-\hat{\omega}_{r}$ 


$$
e=X-\hat{X}=\left[\begin{array}{llll}
e_{I_{s \alpha}} & e_{I_{s \beta}} & e_{\psi_{r \alpha}} & e_{\psi_{r \beta}}
\end{array}\right]^{T}
$$

Now consider the following Lyapunov function:

$$
V=e^{T} e+\frac{\left(\Delta \omega_{r}\right)^{2}}{\lambda}
$$

Its derivative with respect to time is:

$$
\begin{aligned}
& \frac{d V}{d t}=\left\{\frac{d(e)^{T}}{d t}\right\} e+e^{T}\left\{\frac{d e}{d t}\right\}+\frac{1}{\lambda} \frac{d}{d t}\left(\Delta \omega_{r}\right)^{2} \\
\frac{d V}{d t}=e^{T}\left\{(A-K . C)^{T}+(A-K . C)\right\} e & \\
& -2 a_{3} \Delta \omega_{r} \cdot\left(e_{I_{s \alpha}} . \hat{\varphi}_{r \beta}-e_{I_{s \beta}} . \hat{\varphi}_{r \alpha}\right)+\frac{2}{\lambda} \Delta \omega_{r} \frac{d}{d t} \hat{\omega}_{r}
\end{aligned}
$$

A sufficient condition for uniform asymptotic stability is that equation (34) is negative, which amounts to cancel the last two terms in this equation (since the other terms of the second member of (27) are always negative), in which case we can deduce the adaptation law to estimate the rotor speed by equating the second and third term of Eq.

It is estimated the speed by a PI controller described by the relationship:

$$
\hat{\omega}_{r}=K_{p}\left(e_{I_{s \alpha}} \cdot \hat{\varphi}_{r \beta}-e_{I_{s \beta}} \cdot \hat{\varphi}_{r \alpha}\right)+\frac{K_{i}}{s} \int\left(e_{I_{s \alpha}} \cdot \hat{\varphi}_{r \beta}-e_{I_{s \beta}} \cdot \hat{\varphi}_{r \alpha}\right) d t
$$

With $\mathrm{Kp}$ and $\mathrm{Ki}$ are positive constants.

\subsection{Adaptive Luenberger Observer for Speed and Stator Resistance Estimation}

Vector control is sensitive to the motor parameter variation. Especially, stator and rotor resistance vary widely with the motor temperature, [5], [6].

If the rotor speed and stator resistance are considered as variable parameters, assuming no other parameter variations, so the state space of the observer becomes as follows:

$$
\hat{\dot{X}}=\left(A_{\omega_{r}}\left(\hat{\omega}_{r}\right)+A_{R_{s}}\left(\hat{R}_{s}\right)\right) \cdot \hat{X}+B \cdot U+K \cdot\left(I_{s}-\hat{I}_{s}\right)
$$

With $\left(I_{s}-\hat{I}_{s}\right)=\left[\begin{array}{ll}I_{s \alpha}-\hat{I}_{s} & I_{s \beta}-\hat{I}_{s}\end{array}\right]$

The estimation error of the stator current and rotor flux is given by:

$$
\dot{e}=(A-K \cdot C) \cdot e+\left[(\Delta A)+\left(\Delta A^{\prime}\right)\right] \cdot \hat{X}
$$

With:

$$
\Delta A^{\prime}=\left[\begin{array}{cccc}
-a_{6} \cdot \Delta R_{s} & 0 & 0 & 0 \\
0 & -a_{6} \cdot \Delta R_{s} & 0 & 0 \\
0 & 0 & 0 & 0 \\
0 & 0 & 0 & 0
\end{array}\right]
$$

A Lyapunov function candidate is defined as follows:

$$
V^{\prime}=e^{T} e+\frac{\left(\Delta \omega_{r}\right)^{2}}{\lambda}+\frac{\left(\Delta R_{s}\right)^{2}}{\lambda^{\prime}}=V+\frac{\left(\Delta R_{s}\right)^{2}}{\lambda^{\prime}}
$$

And

$$
\Delta \omega_{r}=\omega_{r}-\hat{\omega}_{r} ; \Delta R_{S}=R_{S}-\hat{R}_{S}
$$

The adaptive scheme for stator resistance estimation is found by, [5], [6]:

$$
\begin{aligned}
& \hat{R}_{s}=K_{p}\left(\left(i_{s \alpha}-\hat{i}_{s \alpha}\right) \cdot \hat{i}_{s \alpha}+\left(i_{s \beta}-\hat{i}_{s \beta}\right) \cdot \hat{i}_{s \beta}\right)+ \\
& \frac{K_{i}}{s} \int\left(\left(i_{s \alpha}-\hat{i}_{s \alpha}\right) \cdot \hat{i}_{s \alpha}+\left(i_{s \beta}-\hat{i}_{s \beta}\right) \cdot \hat{i}_{s \beta}\right) d t \\
& \hat{R}_{s}=K_{p}\left(e_{i_{s \alpha}} \cdot \hat{i}_{s \alpha}+e_{i_{s \beta}} \cdot \hat{i}_{s \beta}\right)+\frac{K_{i}}{s} \int\left(e_{i_{s \alpha}} \cdot \hat{i}_{s \alpha}+e_{i_{s \beta}} \cdot \hat{i}_{s \beta}\right) d t
\end{aligned}
$$

With:

$$
i_{s \alpha}-\hat{i}_{s \alpha}=e_{i_{s \alpha}} ; i_{s \beta}-\hat{i}_{s \beta}=e_{i_{s \beta}}
$$

$\mathrm{Kp}$ and $\mathrm{Ki}$ are positive constants. The role of adaptive mechanisms is to minimize the following errors.

\subsection{Estimation of Rotor Resistance}

To improve the performance and simplify the vector control and reduce its cost, we will base this part on a hypothesis to deduce the value of the rotor resistance estimate from the estimation of the stator resistance. Still, it is assumed that the motor windings are almost at the same temperature, and neglecting the skin effect, the resistances will vary proportionally.

The rotor resistance estimate can be determined by the following relation, [9], [10]:

$$
\hat{R}_{r}=\hat{R}_{s} \cdot \frac{R_{r . n}}{R_{s . n}}
$$




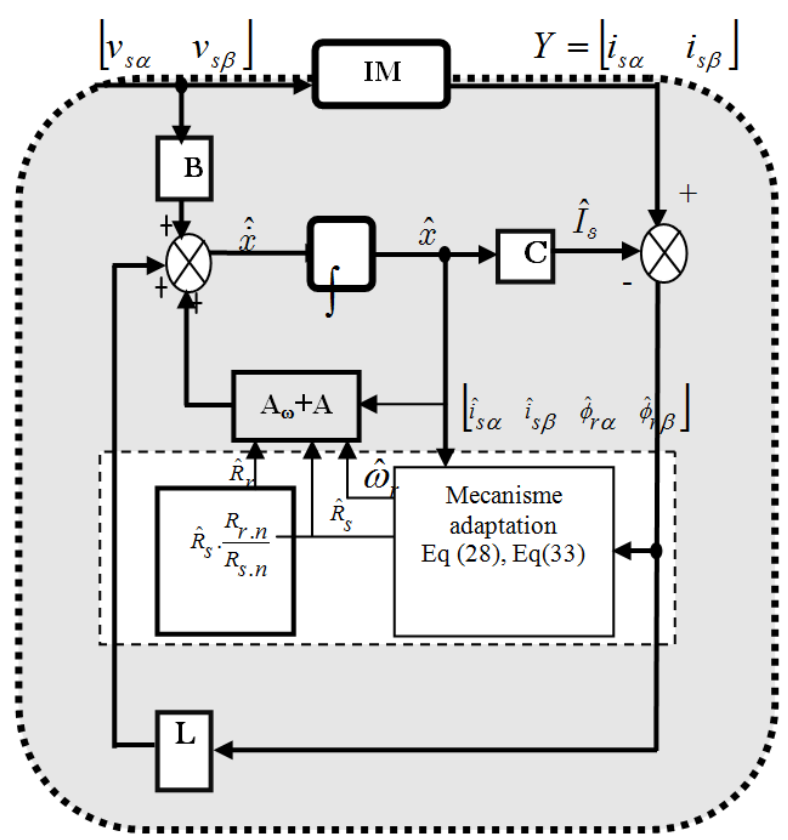

Figure 3. Luenberger Observer for simultaneous estimation of speed, $R_{r}$ and $R_{s}$.
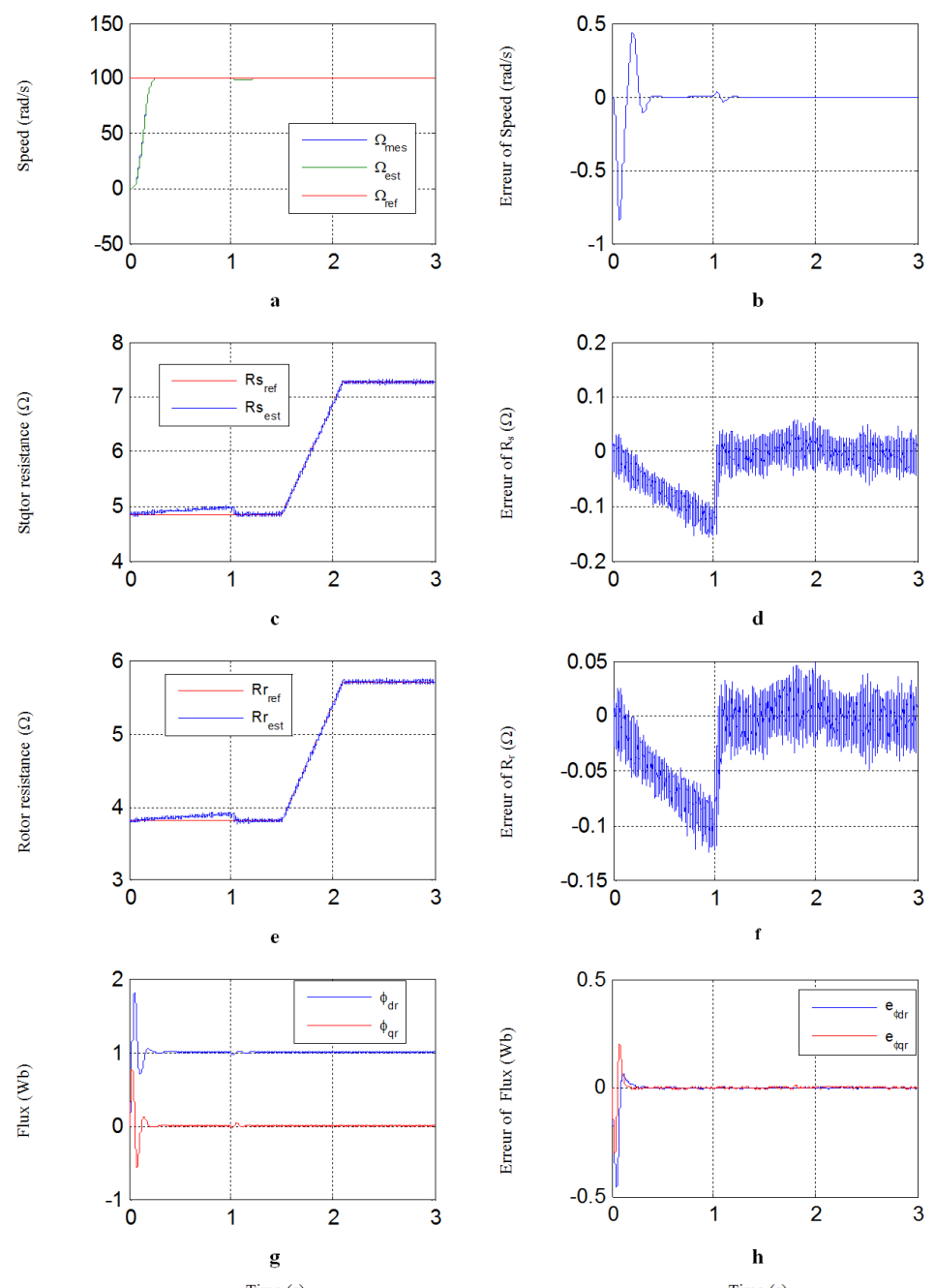

Figure 4. Simulation results of the speed estimation with stator resistance increased sharply by $50 \%$ from Rs and rotor resistance increased sharply by $50 \%$ from $R r$. 

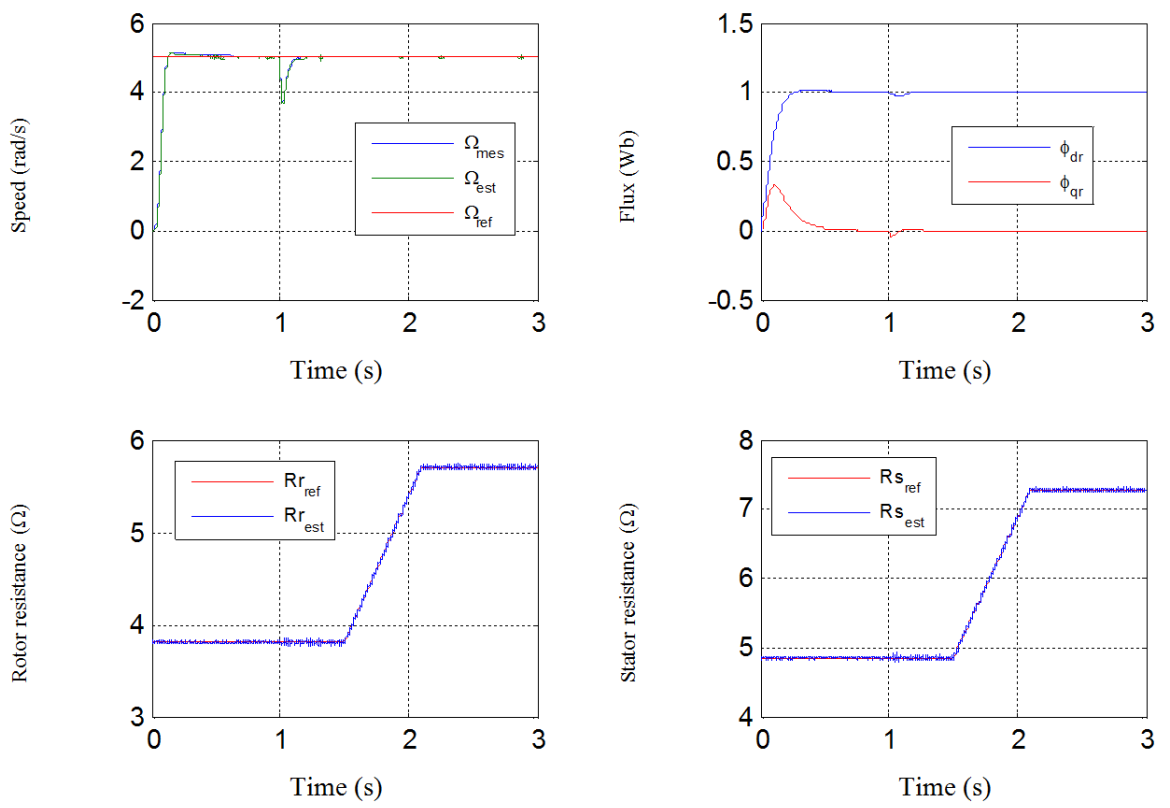

Figure 5. Speed sensorless control with stator and rotor resistance estimation at low speed.

\section{Results of Simulations and Discussion}

Adjustment performance with the proposed observation algorithm is evaluated by numerical simulation for several operating conditions of the machine with indirect vector control (IFOC).

The simulation results (Figures 4), show the simultaneous estimation of the speed and two resistors (stator and rotor)

It is clear that:

The estimated resistances converge to the nominal resistances quickly and with great accuracy, or the estimation error is acceptable after a very short transient regime.

The injection of these values into the flux observer keeps the performances of the flux observer and the vector control, in fact all the basic magnitudes of the machine (speed, rotor flux, stator currents, and the torque) converge to their nominal values.

So can say that all the parameters are identical (resistances of the motor and the observer).

Figure 5 illustrates simulation results of Speed Sensorless Vector Control based on speeed estimation and two resistors simultaneously, for a low speed reference $(5 \mathrm{rad} / \mathrm{sec})$ and application of a $10 \mathrm{Nm}$ resistive torque at times $\mathrm{t}=1 \mathrm{~s}$.

From these results, find that:

At the beginning of the simulation the stator resistance is equal to its nominal value. At time $t=1.5 \mathrm{sec}$ the value of Rs increases to a value of $7.275 \mathrm{Ohm}$, which corresponds to an increase of $+50 \%$ of its nominal value as well as the rotor resistance $(+50 \% \mathrm{Rr})$.

So, can note that:

- The estimation of the two resistances carried out precisely,

- The estimation of the speed is done correctly

- The decoupling is still maintained, which proves the efficiency of the rotor resistance estimation
This proves that our system responds successfully to this type of test and the estimator remains stable.

\section{Conclusion}

This paper has outlined a new scheme for a sensorless speed control scheme for induction motors. Theoretical analysis and simulation results demonstrate that the proposed speed control scheme has a good speed response and so confirm the feasibility of the proposed algorithm. Practical implementation of the proposed method is a subject of future follow up research work.

\section{Appendix}

Induction Motor Parameters

$50 \mathrm{~Hz}, 1.5 \mathrm{Kw}, 1420 \mathrm{rpm}, 380 \mathrm{~V}, 3.7 \mathrm{~A}, \mathrm{Rr}=3.805 \Omega$, $\mathrm{Rs}=4.85 \Omega$, Ls $274=\mathrm{mH}$, Ls $274=\mathrm{mH} \quad 0.031=\mathrm{J} \quad \mathrm{kg} . \mathrm{m}^{2}$, $\mathrm{F}=0.00114 \mathrm{~kg} \cdot \mathrm{m}^{2} / \mathrm{s}$

\section{References}

[1] Y. Agrebi Zorgani, Y. Koubaa, M. Boussak, "Simultaneous Estimation of Speed and Rotor Resistance in Sensorless ISFOC Induction Motor Drive Based on MRAS Scheme", XIX International Conference on Electrical Machines - ICEM 2010, Rome.

[2] DJ. CHERIFI, Y. MILOUD, "New Fuzzy Luenberger Observer for Performance Evaluation of a Sensorless Induction Motor Drive", International Review of Automatic Control, Theory and Applications (IREACO), July 2013 (Vol. 6 N. 4)

[3] Yali Zhou, Yongdon Li and Zedong Zheng, "Research of Speed sensorless Vector Control of an Induction Motor Based on Model Reference Adaptive System" 2009 IEEE. 
[4] Juraj Gacho, Milan Zalman, "IM Based Speed Servodrive With Luenberger Observer", Journal of Electrical Engineering, VOL. 61, NO. 3, 2010, 149-156.

[5] Mohamed S. Zaky, "Stability Analysis of Simultaneous Estimation of Speed and Stator Resistance for Sensorless Induction Motor Drives", Proceedings of the 14th International Middle East Power Systems Conference (MEPCON'10), Cairo University, Egypt, December 19-21, 2010, Paper ID 180.

[6] Yehia S. Mohamed, A. M. El-Sawy and A. A. Zaki, "STATOR RESISTANCE ESTIMATION FOR SPEED SENSORLESS VECTOR CONTROLLED INDUCTION MOTOR DRIVES AS INFLUENCED BY SATURATION", Journal of Engineering Sciences, Assiut University, Vol. 37, No. 3, pp. 669-690, May 2009.

[7] M. Messaoudi, L. Sbita, M. Ben Hamed and H. Kraiem, "MRAS and Luenberger Observer Based Sensorless Indirect
Vector Control of Induction Motors", Assain Journal of Information Technology 7 (5): 232-239, 2008.

[8] Mabrouk Jouili, Kamel Jarray, Yassine Koubaa and Mohamed Boussak, "A Luenberger State Observer for Simultaneous Estimation of Speed and Rotor Resistance in sensorless Indirect Stator Flux Orientation Control of Induction Motor Drive", IJCSI International Journal of Computer Science Issues, Vol. 8, Issue 6, No 3, November 2011.

[9] H. Kubota, K. Matsuse, and T. Nakano, "DSP-based speed adaptive flux observer of induction motor," IEEE Trans. Ind. Appl., vol. 29, no. 2, pp. 344-348, Mar./Apr. 1993.

[10] S. M. Nayeem Hasan and Iqbal Husain, "A LuenbergerSliding Mode Observer for Online Parameter Estimation and Adaptation in High-Performance Induction Motor Drives", IEEE Transactions on industry applications, vol. 45, no. 2, March/April 2009. 